Service social

\title{
Problèmes sociaux et service social
}

\section{Robert Mayer, Marcelle Laforest et Jocelyn Lindsay}

Volume 39, numéro 2, 1990

Les problèmes sociaux

URI : https://id.erudit.org/iderudit/706474ar

DOI : https://doi.org/10.7202/706474ar

Aller au sommaire du numéro

Éditeur(s)

École de service social de l'Université Laval

ISSN

1708-1734 (numérique)

Découvrir la revue

Citer ce document

Mayer, R., Laforest, M. \& Lindsay, J. (1990). Problèmes sociaux et service social.

Service social, 39(2), 5-12. https://doi.org/10.7202/706474ar d'utilisation que vous pouvez consulter en ligne.

https://apropos.erudit.org/fr/usagers/politique-dutilisation/ 


\section{AVANT-PROPOS}

\section{Problèmes sociaux et service social}

Pendant longtemps les écrits ainsi que les cours sur les problèmes sociaux ont été une sorte de "fourre-tout » où l'on jetait pêle-mêle tous les problèmes possibles (allant de la guerre nucléaire à la multiplication des animaux dans les villes, en passant par les problèmes de l'obésité, des émeutes raciales et des drogues, pour finalement terminer avec la pornographie et la maladie mentale). C'est un peu caricatural, mais il suffit d'ouvrir un des nombreux recueils de textes ( readers ») sur le sujet pour constater que nous exagérons à peine. Quoi qu'il en soit, ces cours sur les problèmes sociaux ont évolué et ils sont maintenant intégrés dans la majorité des programmes de service social au Québec. Toutefois, le thème de I'analyse des problèmes sociaux, malgré sa position centrale dans la formation des intervenants sociaux, n'a pas fait encore l'objet, curieusement, d'un traitement un peu systématique dans les revues québécoises de service social. II nous a semblé qu'il était temps de tenter de pallier à cette lacune.

C.W. Mills (1968) a bien fait ressortir les principales critiques visà-vis la documentation traditionnelle sur les problèmes sociaux. II souligne que ces volumes sont caractérisés par un très bas degré d'abstraction, à un point tel qu'il est souvent difficile de comprendre en quoi les différents textes, et les problèmes qu'ils veulent analyser, sont reliés les uns aux autres. Le caractère informationnel et descriptif de ces études est relié à la difficulté, pour ne pas dire à l'incapacité des auteurs, de considérer le niveau des structures sociales. Les explications les plus fréquentes des problèmes sociaux sont celles qui font appel aux pulsions biologiques qui suscitent la transgression des normes sociales. On met l'accent sur tout ce qui tend vers un équilibre harmonieux. D'une façon générale, ces auteurs voient les problèmes purement au niveau individuel : ils ont une nette tendance vers le "psychologisme ", d'où l'insistance également sur l'idée d'ajustement. Pour eux, le changement social est souvent perçu comme source principale des problèmes sociaux. Devant l'ampleur des problèmes actuels, il fallait donc s'efforcer de changer de cap. 
D'entrée de jeu, il faut souligner que dans l'usage courant la notion de problème social demeure encore, et ce, malgré sa simplicité apparente, quelque peu imprécise. D'où l'importance de présenter quelques idées maîtresses qui sont relativement simples et qui ont présidé à l'élaboration de ce numéro. Il y a d'abord l'idée de la nécessité d'une certaine réflexion théorique pour bien préciser le concept de problème social et baliser les principales approches analytiques des problèmes sociaux. Cette réflexion devrait nous permettre de mieux saisir le caractère idéologique des écrits sur les problèmes sociaux ainsi que le caractère éminemment relatif de la notion de problème social. Ensuite, il y a nécessité d'élaborer une perspective à la fois historique, comparative et prospective dans I'analyse des problèmes sociaux.

Il importe par ailleurs, de souligner la nécessité de rompre avec un certain nombre de postulats ou d'orientations analytiques qui ne nous apparaissent pas avoir servi la cause de l'intervention sociale, dans la mesure où ces orientations obscurcissent l'analyse des problèmes sociaux plutôt que de l'éclairer. C'est pourquoi nous estimons qu'il faut en finir avec l'analyse idéaliste des problèmes sociaux et avec le processus de "blâmer la victime " (Ryan, 1971). Rappelons qu'un des éléments principaux de l'explication idéaliste est d'attribuer à certaines caractéristiques des individus les causes de leur sort. D'ailleurs, on a souvent reproché aux publications dans le domaine du service social d'être dominées, encore de nos jours, par cet idéalisme traditionnel qui consiste à mettre l'accent sur les valeurs dans I'analyse des problèmes sociaux (Pizzaro, 1969). On peut sans doute arguer que le service social n'est pas seul en cause et qu'il est lui-même tributaire de tout un courant des sciences humaines, principalement celui de la sociologie fonctionnaliste américaine; soit, mais le problème reste le même. II nous faut donc apprendre à nous distancier de cette approche strictement subjectiviste et idéaliste des phénomènes sociaux. Comment ? A. Prigoff (1980) propose une piste. Celle-ci observe que l'enseignement du service social aux États-Unis a permis de préparer les étudiants à reconnaître les problèmes sociaux tant chez les individus que chez les petits groupes et à proposer des changements sans toutefois tenir compte des facteurs extérieurs. Cette perspective a eu pour principal effet de détourner l'attention des intervenants des structures sociales qui créent ces problèmes. Afin d'éviter de perpétuer cette situation de myopie sociale, il faut, selon l'auteure, effectuer une analyse de classe en vue d'abord de conceptualiser, puis ensuite observer et démontrer les relations entre les situations socio-économiques et les différences de succès ou $d^{\prime}$ insuccès social. 
Plus près de nous, plusieurs auteurs ont montré que la gestion des problèmes sociaux se fait de plus en plus en fonction du modèle des populations cibles. Par exemple, pour R. Castel (1981 : 127), la "gestion prévisionnelle des profils humains est désormais réalisable avec l'informatique. II devient ainsi possible « de constituer des flux de population selon n'importe quel critère de différenciation, en particulier les anomalies physiques ou psychiques, les risques dus à l'environnement, les carences familiales, etc. » (1981 : 131). Désormais, le savoir médico-psychologique devient un instrument central pour l'élaboration d'une " politique de gestion différentielle des populations» $(1981: 115)$ et il propose de nouvelles stratégies pour prendre en charge ces populations dites « à risques". R. Castel (1983) a aussi précisé que les nouvelles stratégies de prise en charge des problèmes sociaux ne sont plus uniquement développées dans le prolongement des pratiques curatives assistantialistes ou répressives antérieures. Au contraire, la notion de risque, définie statistiquement par la méthode de l'épidémiologie, devient centrale (1983 : 123). Cette nouvelle stratégie donne priorité à la gestion administrative des populations par rapport aux interventions sur le terrain, ainsi qu'aux activités de dépistage et de surveillance systématique par rapport aux activités traditionnelles d'assistance et de protection (1983: 126).

Récemment M. Drolet (1989) a montré que le Québec n'échappe pas à cette évolution ni à ces débats. En effet, à partir de plusieurs rapports gouvernementaux (Rapport Brunet, Rapport Rochon, etc.) "il se dessine donc divers niveaux de vulnérabilité : allant des personnes ciblées susceptibles d'avoir d'importants problèmes sociaux et de santé, jusqu'à des populations perçues comme potentiellement à risque "(1989:49). Mais plus fondamentalement, elle montre que ce débat, surtout en CLSC, s'inscrit autour de deux pôles, soit le pôle technocratique et le pôle participatif. Le premier, défendu par les principaux centres de décision du réseau sociosanitaire "vise à gérer les problématiques sociales, selon une perspective " comptable » [...] à " coordonner » les ressources et les forces du milieu [...] en fonction de la planification stratégique établie à partir de critères bureaucratiques. Celles-ci apparaissent alors prioritairement comme des ressources disponibles pour répondre aux besoins identifiés par le système public de la clientèle qu'il a aussi déterminée » (1989: 51). Le second est plutôt véhiculé par les intervenants sociaux, les groupes communautaires et les usagers : " II vise essentiellement le respect de l'autonomie des réseaux d'aide formelle et informelle de la communauté, allant de l'autodétermination des ressources communautaires jusqu'à la prise en considération des limites des familles à affronter certaines problématiques. En raison de leur ampleur, les 
problèmes sociaux exigeraient une protection ou un support supplémentaire de l'État. De par ces positions, le pôle participatif s'inscrit donc en opposition au courant technocratique et à la gestion stratégique des systèmes sociaux » (Idem). Sans vouloir aborder tous les éléments de cette problématique, nous allons en explorer quelques-uns.

Pour introduire la question, le texte de R. Mayer et M. Laforest, de nature théorique, aborde la définition du concept de problème social ainsi qu'une présentation des principaux courants analytiques ou courants de pensée qui prévalent dans l'analyse sociologique des problèmes sociaux. Les auteurs présentent tour à tour : l'École de Chicago, le modèle culturaliste, l'approche fonctionnaliste, l'approche critique du conflit social, l'interactionnisme symbolique et les théories de la réaction sociale et de l'étiquetage et, finalement, le constructivisme. Sans prétendre encadrer tous les textes qui suivent, soulignons que plusieurs d'entre eux se réfèrent, plus ou moins explicitement, à l'un ou l'autre de ces courants de pensée.

Ensuite $\mathrm{H}$. Dorvil aborde la maladie mentale comme problème social. L'auteur adopte un point de vue social et s'oppose à une analyse trop exclusivement biologique du phénomène en question. À l'aide de multiples exemples, tant historiques que contemporains, il s'efforce de démontrer que ce sont les normes et les valeurs "qui régissent la construction de l'anormalité dans une collectivité »et qu'en conséquence, la déviance qu'implique ce type de comportement est bel et bien "un problème social avant d'être une maladie ". Il souligne aussi que la perception et la définition de la déviance évoluent dans le temps et l'espace. Finalement, il situe l'analyse du phénomène de la maladie mentale par rapport aux principales théories explicatives des problèmes sociaux et il rappelle les disparités dans la répartition des troubles mentaux selon les classes sociales.

Le troisième texte, rédigé par $P$. Simard, décrit les conditions de vie des itinérants de Montréal. Le thème de l'itinérance n'est certes pas nouveau, mais au cours des dernières années, et surtout dans les grandes villes d'Amérique du Nord, il est réapparu avec une acuité particulière. On peut facilement, lors de nos déplacements quotidiens, observer des personnes itinérantes, mais qui sont-elles vraiment ? Cet article tente d'y répondre, en abordant, tour à tour, divers aspects du phénomène : le profil démographique des itinérants, les racines familiales, les expériences avec les institutions scolaires et sociosanitaires; les expériences de travail et surtout de chômage; la vie de couple et les séparations; le passage de la chambre à la rue, en passant par les refuges, tout en s'attachant à la fois aux conditions objectives de vie (la misère et la maladie) et aux 
conditions plus subjectives (la désorganisation psychosociale, la toxicomanie, la folie), pour finalement nous rappeler que " l'itinérant n'est pas né itinérant » et qu'en conséquence, il est important de comprendre le processus qui conduit vers la clochardise.

À l'aide d'une recension des écrits et d'une enquête menée auprès d'informateurs-clés, C. Chamberland et C. Bouchard abordent le problème de l'incidence des conduites abusives et négligentes de parents envers leurs enfants selon les caractéristiques socioéconomiques des familles. Suite à de nombreuses études descriptives et épidémiologiques, on a tenté de prévoir le problème à l'aide de divers indicateurs ou variables de prédiction. Toutefois, plusieurs milieux échappent à ce modèle de prédiction : "On retrouve des taux de mauvais traitements étonnamment bas dans des secteurs à un très haut niveau de pauvreté; dans d'autres cas, les taux sont anormalement élevés". C'est donc pour mieux comprendre ce phénomène qu'on a mené une enquête "dans le but de fournir un portrait détaillé des caractéristiques physiques et sociales de ces voisinages telles que perçues par une soixantaine d'informateursclés".

Le texte de G. Girardi, traduit de l'italien par G. Doré, aborde le problème de la drogue dans une perspective sociologique, qui met en relation des facteurs structurels et des facteurs culturels. L'auteur part du constat qu'en Italie comme ailleurs dans le monde, il existe un sentiment d'échec et d'impuissance généralisé face au "fléau de la drogue ». Les diverses pistes de solution proposées le plus souvent par les gouvernements "se sont révélées non seulement inefficaces mais souvent contre-productives ». Devant cet état de fait, l'auteur avance l'hypothèse que le phénomène de la drogue, tant au niveau de la distribution (trafic) qu'à celui de la consommation, "plonge ses racines dans la logique même du système capitaliste, dans ses structures et dans sa culture; c'est pourquoi la société ne peut affronter sérieusement le problème sans se remettre elle-même en cause ". Aussi, l'amorce de solution à ce problème passe-t-elle nécessairement par l'élaboration d'un projet de société. Pour l'auteur, la toxicomanie est certes une déviance; " mais beaucoup plus profonde et mortelle est la déviance qu'elle dénonce, celle d'un système qui confisque la richesse, le pouvoir, la culture, la vie, au bénéfice d'une minorité en excluant de grandes masses d'hommes et de femmes".

Le texte de $S$. Jutras et $M$. Renaud présente les résultats d'un vaste sondage $d^{\prime}$ opinions concernant des attitudes générales en matière de problèmes sociaux ou sociosanitaires et plus particulièrement par rapport à deux situations-problèmes : devoir prendre en charge un parent âgé en perte d'autonomie ou devoir assumer les respon- 
sabilités de chef de famille monoparentale. Mais en plus de l'analyse de ces deux problèmes spécifiques, cette enquête nous fournit de précieuses informations sur les types de solutions concrètes qu'une population se donne pour faire face aux divers problèmes sociaux qui la confrontent.

$R$. Lefrançois traite de la recherche sociale sur les problèmes sociaux. II note que la conjoncture du début des années 1980 a eu son effet sur la perception et la définition des problèmes sociaux : " Des conceptions renouvelées de l'intervention et des problèmes sociaux ont vu le jour, marquées du sceau de l'individualisme et sous l'impulsion de l'idéologie néo-conservatrice qui pénètre dorénavant nos modes de vie ». Pour l'auteur, la résurgence des modèles explicatifs psychologisants de même que la préoccupation pour les problèmes reliés à l'adaptation ou à l'insertion sociale des individus risque de signifier un retour en arrière. De même, les politiques sociales de maintien à domicile et de désinstitutionnalisation signifient qu'une partie de la responsabilité de solutionner les problèmes sociaux est confiée au réseau communautaire, alors que l'État se désengage progressivement du champ social. Tout cela risque d'avoir un effet sur la recherche sociale, et notamment sur la façon dont les chercheurs se représentent les problèmes sociaux. Pour tenter d'éclairer cette question, l'auteur a réalisé une enquête auprès de chercheurs du Québec associés à la recherche sociale appliquée (tant des milieux universitaires que du réseau des affaires sociales). Dans I'analyse qui accompagne la présentation des données de cette étude, il constate que la recherche produit actuellement des connaissances "soit trop générales ou descriptives, soit des connaissances hyperspécialisées et fragmentées ", empêchant ainsi l'élaboration d'un savoir intégré sur les problèmes sociaux. Tout cela amène l'auteur à conclure que même au lendemain du Rapport Rochon, le débat sur le rôle et la place de la recherche sociale en matière d'analyse et de solution aux problèmes sociaux est encore à faire.

A. Beaudoin, pour sa part, analyse la conception des problèmes sociaux qui se dégage du Rapport de la Commission d'enquête sur les services de santé et les services sociaux (Commission Rochon) et les conséquences pour l'élaboration de l'action sociale et de la politique sociale. L'auteur part d'une distinction entre les problèmes sociétaux, qui sont d'ordre structurel, et les problèmes sociaux, ceux qui sont associés à des personnes ayant des caractéristiques spécifiques ou à des groupes sociaux. Ensuite, il montre comment la Commission, tout en prenant acte des multiples changements structurels qui ont caractérisé le Québec des dernières années (c'est-à-dire les problèmes sociétaux) s'oriente par la suite vers une analyse des problèmes sociaux. L'auteur situe la place de la pauvreté dans 
I'analyse développée par la Commission. II explique pourquoi la Commission s'est orientée vers l'identification de groupes cibles : jeunes, familles monoparentales et personnes seules. Finalement, il résume l'analyse de la Commission pour ce qui est de l'identification des principaux problèmes de santé et des problèmes sociaux.

Dans la section "Commentaires et documents », nous avons également retenu un certain nombre de textes reliés plus ou moins directement au thème de ce numéro. Ainsi, $D$. Fortin fait le lien entre problèmes sociaux et politiques sociales dans la mesure où il procède à une analyse critique de la récente réforme de l'aide sociale au Québec. Après avoir rappelé le contexte socio-économique (crise économique au début des années 1980, crise de l'État-Providence, etc.), l'auteur estime que cette réforme s'inscrit dans un «virage social ", marqué au coin d'un "néo-conservatisme", ou d'un "Reaganisme social », d'où son caractère punitif vis-à-vis les assistés sociaux définis comme "une bande de paresseux et de fraudeurs". Mais après avoir décrit et commenté les principaux éléments de cette réforme, l'auteur estime que celle-ci risque de produire, par la fameuse alchimie des effets pervers, des résultats contraires à ceux escomptés, soit "l'amplification de l'appauvrissement des bénéficiaires et de l'oppression sociale dont ils sont déjà victimes ". Finalement, D. Fortin montre comment cette réforme s'inscrit au cœur d'un nouveau projet de société.

M. Parazelli présente une réflexion critique sur la stratégie d'encadrement étatique des pratiques communautaires, dans une approche techno-médicale des problèmes sociaux et de ses conséquences sur l'analyse même de ces problèmes. Cette réflexion s'inscrit dans l'analyse de la réforme actuelle des services de santé et des services sociaux. Après avoir présenté les différents aspects de cette réforme, l'auteur fait ressortir l'opposition entre l'approche des problèmes sociaux soutenue par l'État et celle défendue par les organismes communautaires autonomes. Par ailleurs, I'auteur critique le projet de réforme en soulignant notamment ses effets néfastes pour la population en général et en particulier pour les jeunes. II dénonce également l'utilisation en sous-traitance des groupes communautaires par l'État.

L'article de G. Martin et F. Lavoie fait état d'une partie des résultats d'une recherche qualitative sur les comportements d'aide des médecins et des infirmières en matière de violence conjugale. Ces intervenants, au nombre d'une vingtaine, pratiquaient soit en milieu hospitalier, soit en pratique privée. L'analyse des comportements d'aide est regroupée autour de deux axes principaux : I'action directe auprès des femmes violentées et l'action avec les ressources. Par la suite, la discussion des principaux résultats porte 
sur la relation entre intervenant ou intervenante et femme violentée, sur l'éthique reliée à la suggestion de départ du foyer et enfin, sur la contribution de ces intervenants et intervenantes à l'ensemble du système d'aide en faveur des femmes violentées.

Finalement, M. O'Neill et al. procèdent à un résumé-critique sur une série de cinq livres sur la périnatalité au Québec. Ces informations sont susceptibles d'être utiles aux intervenants des services de santé et des services sociaux mais surtout ils éclairent le débat, qui dure maintenant depuis une vingtaine d'années, entre divers acteurs sociaux (médecins, infirmières, sages-femmes, administrateurs) et qui met en lumière " des visions et des approches parfois complémentaires mais souvent opposées quant aux manières de prendre en charge la grossesse, l'accouchement et les premières années de la vie ».

Service social a déjà consacré de nombreux numéros à l'étude de problèmes sociaux, par exemple la pauvreté, la délinquance, le contrôle des naissances, les difficultés de logement ou le vieillissement. Le présent numéro apporte toutefois une contribution spécifique, puisqu'il aborde la nature même des problèmes sociaux et les dimensions théoriques et idéologiques sous-jacentes à leur définition.

\author{
Robert Mayer \\ Marcelle Laforest \\ Jocelyn Lindsay
}

\title{
Références
}

CAStel, R. (1981). La gestion des risques. Paris : Éditions de Minuit.

CASTEL, R. (1983). "De la dangerosité au risque ", Actes de la recherche en sciences sociales, Paris, $\mathrm{n}^{\text {os }}$ 47-48 : 119-127.

DROLET, M. (1989). "La réaliser sans la nommer ? La réorientation des CLSC à travers la notion de risque ", Revue canadienne de politique sociale, vol. $24: 48-58$.

Mills, C.W. (1968). L'imagination sociologique. Paris : F. Maspéro.

Pizzaro, N. (1969). "La notion de groupe marginal : idéologie et réalité", Socialisme, Montréal, juillet-août : 76-86.

Prigoff, A. (1980). "Current Development in Social Work Education in the United States", Revue canadienne d'éducation en service social, vol. 6, $\mathrm{n}^{\circ} 1: 132$.

RYAN, W. (1971). Blaming the Victim. New York : Vintage Books. 